\title{
Management of Acquired Cholesteatoma Associated With Patulous Eustachian Tube and Habitual Sniffing
}

\author{
Sung-Won Choi (i) $\cdot$ Dong-Joo Lee (i] $\cdot$ Seok-Hwan Lee (i) $\cdot$ Se-Joon Oh (i) $\cdot$ Soo-Keun Kong (i) \\ Department of Otorhinolaryngology and Biomedical Research Institute, Pusan National University Hospital, \\ Pusan National University School of Medicine, Busan, Korea
}

Objectives. To investigate the effect of surgical treatment with eustachian tube (ET) catheter insertion in patients with acquired cholesteatoma associated with patulous eustachian tube (PET) and habitual sniffing.

Methods. Nine ears of nine patients (two men and seven women; age, 20 to 65 years; average, $37.9 \pm 12.0$ years) of acquired cholesteatoma associated with PET and habitual sniffing who underwent cholesteatoma surgery with simultaneous additional ET catheter insertion were examined in this study. Successful treatment was defined as stoppage of sniffing, a relief of a PET handicap inventory-10 (PHI-10), an improvement of autophony grade and no cholesteatoma recurrence.

Results. ET catheter insertion was performed in all ears. Follow-up duration ranged from 16 to 37 months (average, 25.4 months). Cases consisted of nine pars flaccida type (100\%). All patients obtained relief from aural symptoms and stopped sniffing. Postoperative PHI-10 scores were significantly lower than preoperative scores $(P<0.001)$. During an average follow-up of 25.4 months, no cholesteatoma recurrence has occurred to date. One patient developed otitis media with effusion (OME) post-catheterization; OME resolved spontaneously without treatment. Four patients had a consecutive ET catheter insertion on the other side to resolve PET-related aural symptoms.

Conclusion. In case of acquired cholesteatoma with PET and habitual sniffing, ET catheter insertion performed simultaneously with cholesteatoma surgery could help reduce aural symptoms and stop sniffing. Moreover, the procedure might help in preventing cholesteatoma recurrence.

Keywords. Cholesteatoma; Patulous Eustachian Tube; Habitual Sniffing

\section{INTRODUCTION}

Patients with a patulous eustachian tube (PET) can develop habitual sniffing to alleviate uncomfortable aural symptoms such as autophony [1]. Several studies have reported that habitual sniffing in patients with PET is closely related to the pathogene-

\footnotetext{
- Received December 19, 2018

Revised February 25, 2019

Accepted March 14, 2019

- Corresponding author: Soo-Keun Kong

Department of Otorhinolaryngology and Biomedical Research Institute, Pusan National University Hospital, Pusan National University School of Medicine, 179 Gudeok-ro, Seo-gu, Busan 49241, Korea

Tel: +82-51-240-7335, Fax: +82-51-246-8668

E-mail: entkong@gmail.com
}

sis of cholesteatoma [2-9]. Its incidence in acquired middle ear cholesteatoma has been reported to be as high as $25.7 \%$ and $27.4 \%$ respectively $[9,10]$. The incidence of eustachian tube (ET) closing failure is much higher $(54.8 \%)$ in cases with pars flaccida retraction cholesteatoma [11]. The middle-ear pressure created by habitual sniffing was considered to be an important initiator of cholesteatoma [12]. In addition, postoperative recurrence of cholesteatoma is significantly associated with continuing habitual sniffing after the surgery [10]. Therefore, it is important that cholesteatoma patients with PET stop habitual sniffing to prevent recurrence. However, the treatment of PET with habitual sniffing is not easy. Several studies have reported that surgical treatment that partially blocks the ET could help PET patients to stop sniffing [13-15]. Though the mechanical or functional

Copyright (C) 2019 by Korean Society of Otorhinolaryngology-Head and Neck Surgery.

This is an open-access article distributed under the terms of the Creative Commons Attribution Non-Commercial License (http://creativecommons.org/licenses/by-nc/4.0)

which permits unrestricted non-commercial use, distribution, and reproduction in any medium, provided the original work is properly cited. 
obstruction of the ET has been regarded as a major factor in the pathogenesis of middle ear diseases including cholesteatoma, this concept has not been widely accepted. In this study, we evaluate the safety and efficacy of additional ET catheter insertion performed simultaneously with cholesteatoma surgery (atticotomy or simple mastoidectomy) in acquired cholesteatoma patients associated with PET and habitual sniffing.

\section{MATERIALS AND METHODS}

We retrospectively analyzed patients with acquired cholesteatoma associated with PET and habitual sniffing, who underwent cholesteatoma surgery with simultaneous additional ET catheter insertion, between January 2015 and December 2017. The diagnosis of PET was based on the presence of aural symptoms such as autophony and/or fullness, decrease in symptoms by closing the ET (supine or prone position, or artificial obstruction of the pharyngeal orifice by maneuvers such as using cotton or jelly) and objective findings of an open ET (tympanic membrane movement upon respiration) $[16,17]$. Only patients who met all three criteria were diagnosed with definite PET and included for analysis. We also assessed the presence of habitual sniffing. Most patients were not conscious of their sniffing habits. The manner in which sniffing was performed to relieve aural symptoms such as aural fullness, autophony, or hyperacusis varied from patient to patient; so, before a simple yes or no question, demonstration of various sniffing actions by a doctor was necessary to help the patients to decide whether or not patients actually had a sniffing habits. The presence of habitual sniffing was confirmed when both uncomfortable aural symptoms (aural fullness, autophony, or hyperacusis) and a history of habitual sniffing to alleviate such aural symptoms were observed [12]. The judgement of habitual sniffing was based on both the habitual occurrence of sniffing and the fact that the patients themselves realized such sniffing was performed to relieve symptoms such as aural fullness, hyperacusis and autophony. Sniffing for nasal diseases such as allergic rhinitis and chronic sinusitis was excluded from this study. Standard tonal audiometry for air $(125,250,500,1,000,2,000$, $4,000$, and $8,000 \mathrm{~Hz})$ and bone conduction $(250,500,1,000$,

\section{H I G G L I G GHTIS}

- Eustachian tube catheter insertion performed simultaneously with cholesteatoma surgery could be helpful to reduce aural symptoms and stop habitual sniffing.

- Eustachian tube catheter insertion performed simultaneously with cholesteatoma surgery might help in preventing cholesteatoma recurrence.

- The presence of habitual sniffing and patulous eustachian tube must be evaluated before cholesteatoma surgery.
2,000, and 4,000 Hz) was performed before and after surgery.

The patients were examined using a microscope and an endoscope for recurrence of postoperative cholesteatoma. Diffusionweighted magnetic resonance imaging was performed in suspicious cases. The patient's self-reporting of presence or absence of autophony was used as the outcome measure as (1) complete relief; (2) significant improvement, satisfied; (3) significant improvement, dissatisfied; (4) unchanged; and (5) worse [18]. The continuity of postoperative habitual sniffing was assessed in the same manner before surgery. To evaluate PET symptoms during the preoperative and postoperative periods, a PET handicap inventory-10 (PHI-10) scoring system was also used [16]. The postoperative PHI-10 outcome measurement was defined as: (1) complete relief (0-8 points); (2) significant improvement (10-16 points); (3) slight improvement (18-24 points); (4) unchanged (26-40 points) (Table 1) [19]. The study was approved by Insti-

Table 1. Patulous eustachian tube handicap inventory-10 (PHI-10) questionnaire [19]

\begin{tabular}{|c|c|c|c|c|}
\hline No. & Question & Never & odera & Severe \\
\hline 1 & $\begin{array}{l}\text { Because of your symptom, is it difficult for } \\
\text { you to concentrate? }\end{array}$ & 0 & 2 & 4 \\
\hline 2 & $\begin{array}{l}\text { Does the loudness of your symptom make } \\
\text { it difficult for you to hear people? }\end{array}$ & 0 & 2 & 4 \\
\hline 3 & Does your symptom make you angry? & 0 & 2 & 4 \\
\hline 4 & $\begin{array}{l}\text { Do you feel as though you cannot escape } \\
\text { your symptom? }\end{array}$ & 0 & 2 & 4 \\
\hline 5 & $\begin{array}{l}\text { Does your symptom interfere with your } \\
\text { ability to enjoy social activities? }\end{array}$ & 0 & 2 & 4 \\
\hline 6 & $\begin{array}{l}\text { Because of your symptom, do you feel } \\
\text { frustrated? }\end{array}$ & 0 & 2 & 4 \\
\hline 7 & $\begin{array}{l}\text { Does your symptom interfere with your job } \\
\text { or household responsibilities? }\end{array}$ & 0 & 2 & 4 \\
\hline 8 & $\begin{array}{l}\text { Do you feel that your symptom has replaced } \\
\text { stress on your relationships with members } \\
\text { of your family and friends? }\end{array}$ & 0 & 2 & 4 \\
\hline 9 & $\begin{array}{l}\text { Do you find it difficult to focus your attention } \\
\text { away from your symptom and on to other } \\
\text { things? }\end{array}$ & 0 & 2 & 4 \\
\hline 10 & Does your symptom make you feel anxious? & 0 & 2 & 4 \\
\hline
\end{tabular}

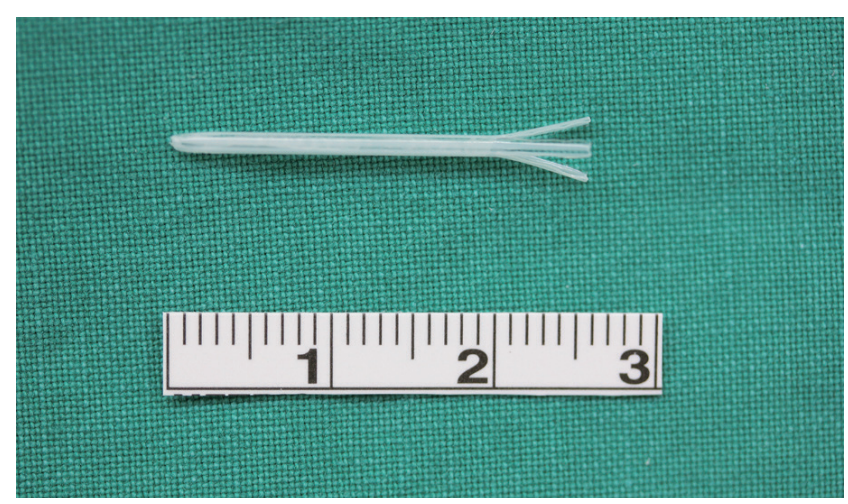

Fig. 1. Image of bone wax filled "tripod"-shaped catheter. 
tutional Review Board of Pusan National University Hospital (IRB No. H-1712-012-062) and informed consent was waived.

\section{Surgical technique}

All patients underwent atticotomy or simple mastoidectomy to remove acquired cholesteatoma. Additional ET catheter insertion was performed simultaneously with cholesteatoma surgery. The diameter and length of the ET was variable. We determined the diameter and length of the catheter based on previous studies $[13,14]$. To design the catheter which would be inserted, we

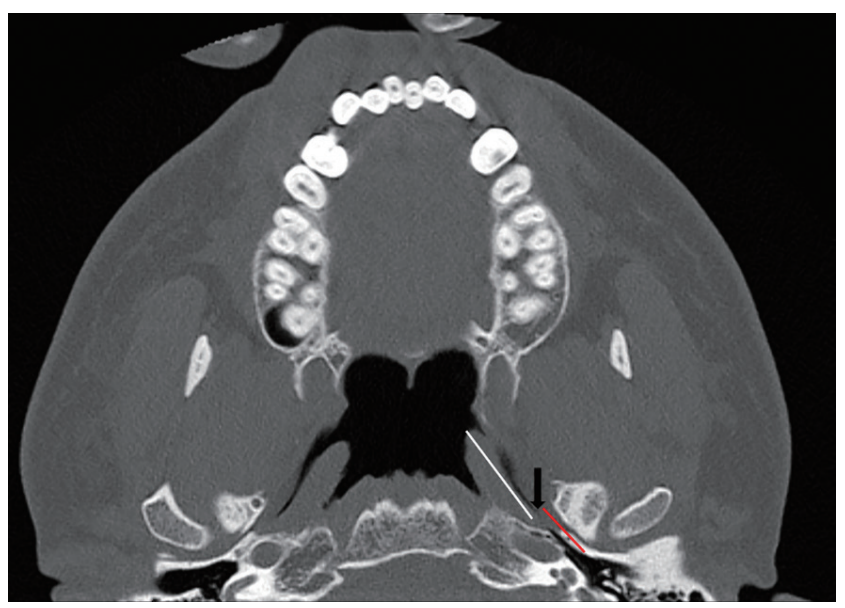

Fig. 2. The estimated length from the isthmus to the bony orifice of eustachian tube (ET) is $16.1 \mathrm{~mm}$ (red line). The estimated length from the isthmus to the nasopharyngeal orifice of ET is $23.2 \mathrm{~mm}$ (white line). Isthmus of ET is located between the dilated visualized segment and the undilated nonvisualized segment of ET (arrow).

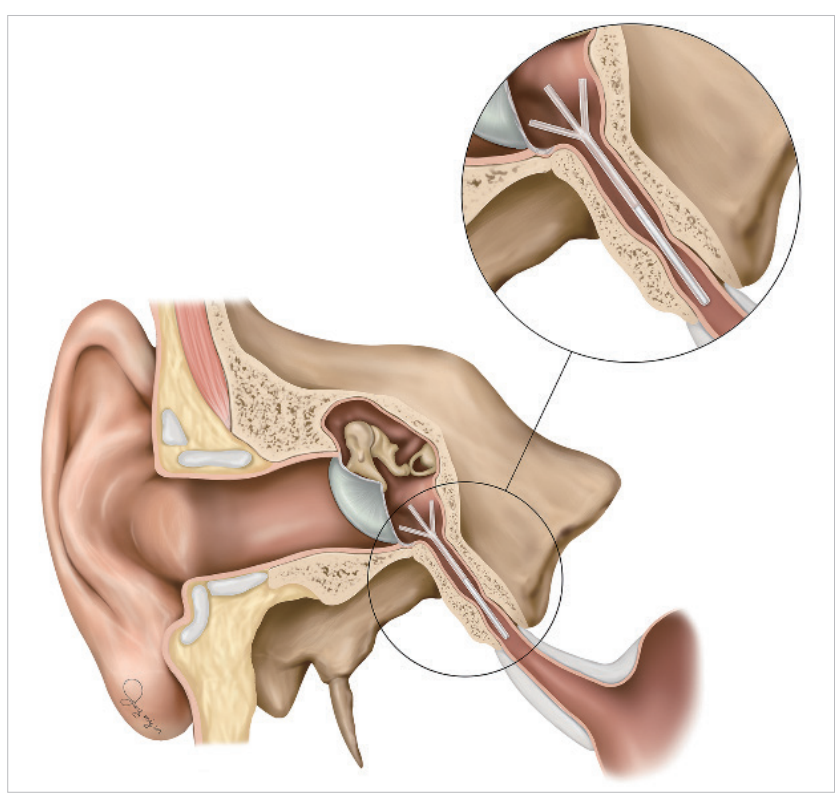

Fig. 3. The length of the catheter was $25 \mathrm{~mm}$. The tip of the catheter passed the isthmus of the eustachian tube and partially obstructed the eustachian tube.

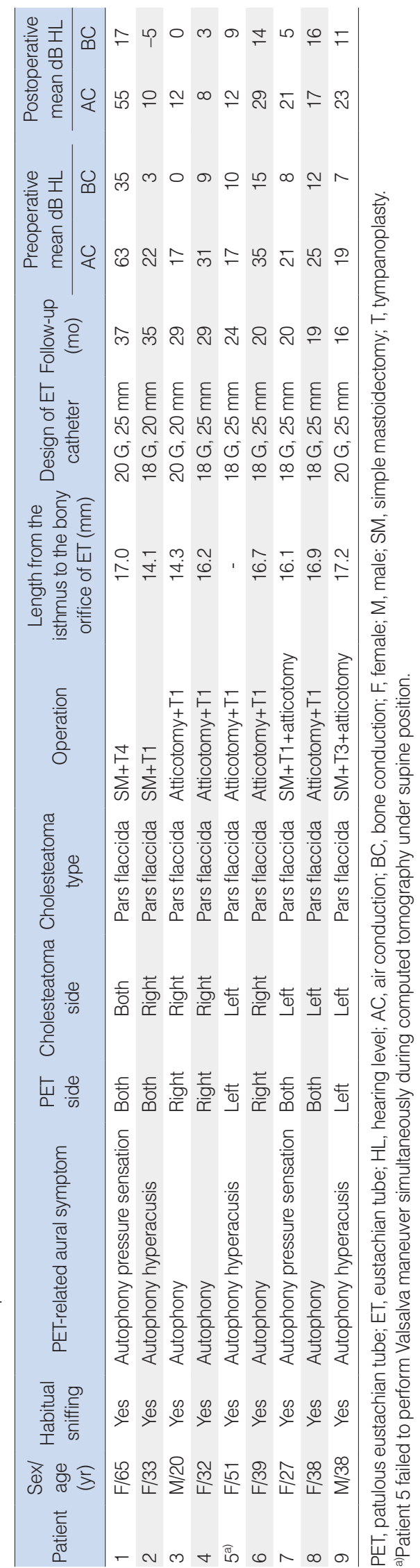


used $18 \mathrm{G}$ (length, $20 \mathrm{~mm}$ or $25 \mathrm{~mm}$; diameter, $1.3 \mathrm{~mm}$ ) and $20 \mathrm{G}$ catheter (length, $20 \mathrm{~mm}$ or $25 \mathrm{~mm}$; diameter, $1.1 \mathrm{~mm}$ ). One end of the catheter was filled with bone-wax, the other end was divided into three sections with a length of $5 \mathrm{~mm}$. Next, we spread the three arms to make a tripod (Fig. 1). The length from the isthmus to the bony orifice of ET was variable. We estimated the length preoperatively by temporal bone computed tomography while the patient was actively performing the Valsalva maneuver simultaneously [20]. Multiplanar reconstruction of the images in the axis of the ET was performed (Fig. 2). The length of the catheter covered the distance from the isthmus to the bony orifice of the ET. Using a $30^{\circ}, 2.7-\mathrm{mm}$ endoscope, the bony orifice of ET was visualized. The catheter tip filled with bone-wax was smoothly inserted into the bony orifice of ET until the resistance of the isthmus was felt (Fig. 3). The placement of catheter was confirmed with endoscopic visualization.

\section{RESULTS}

Additional ET catheter insertion was performed in nine ears of nine patients (two men and seven women; age, 20-65 years; average, $37.9 \pm 12.0$ years) with acquired cholesteatoma associated with PET and habitual sniffing who underwent cholesteatoma surgery (Table 2). In this study, all patients were confirmed PET preoperatively by symptoms go together with directly observing

Table 3. Postoperative outcome measurements

\begin{tabular}{|c|c|c|c|c|c|}
\hline \multirow{2}{*}{ Patient } & \multirow{2}{*}{ Operation } & \multicolumn{2}{|r|}{ Autophony } & \multicolumn{2}{|c|}{ PHI-10 score } \\
\hline & & Preoperative & Postoperative & Preoperative & Postoperative \\
\hline 1 & $\mathrm{SM}+\mathrm{T} 4$ & Presence & Significant improvement, satisfied & 16 & 8 \\
\hline 2 & $\mathrm{SM}+\mathrm{T} 1$ & Presence & Significant improvement, satisfied & 20 & 10 \\
\hline 3 & Atticotomy+T1 & Presence & Complete relief & 10 & 3 \\
\hline 4 & Atticotomy+T1 & Presence & Complete relief & 2 & 0 \\
\hline 5 & Atticotomy+T1 & Presence & Significant improvement, satisfied & 8 & 5 \\
\hline 6 & Atticotomy+T1 & Presence & Significant improvement, satisfied & 12 & 2 \\
\hline 7 & SM+atticotomy+T1 & Presence & Significant improvement, satisfied & 22 & 4 \\
\hline 8 & Atticotomy+T1 & Presence & Significant improvement, satisfied & 16 & 2 \\
\hline 9 & SM+atticotomy+T3 & Presence & Complete relief & 36 & 2 \\
\hline
\end{tabular}

$\mathrm{PHI}-10$, patulous eustachian tube handicap inventory-10; SM, simple mastoidectomy; T, tympanoplasty.
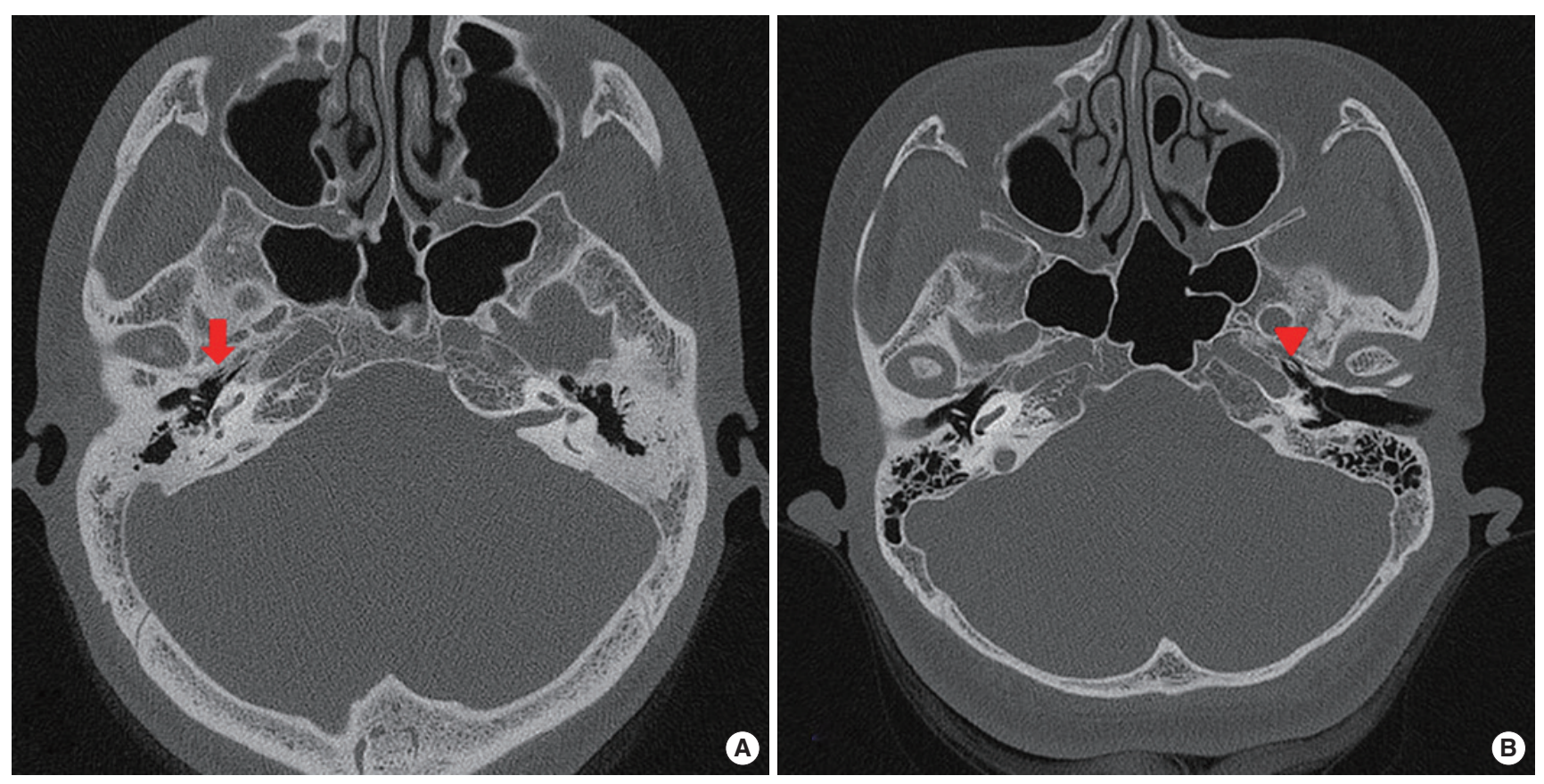

Fig. 4. (A) Temporal bone computed tomography 3 months after atticotomy and tympanoplasty type I with additional eustachian tube catheter insertion (right ear) showing good middle ear aeration and mastoid cavity pneumatization. Catheter (arrow) is located in the bony orifice of the eustachian tube. (B) Temporal bone computed tomography 12 months after atticotomy and tympanoplasty type I with additional eustachian tube catheter insertion (left ear) showing good middle ear aeration. Catheter (arrowhead) is located in the bony orifice of the eustachian tube. 


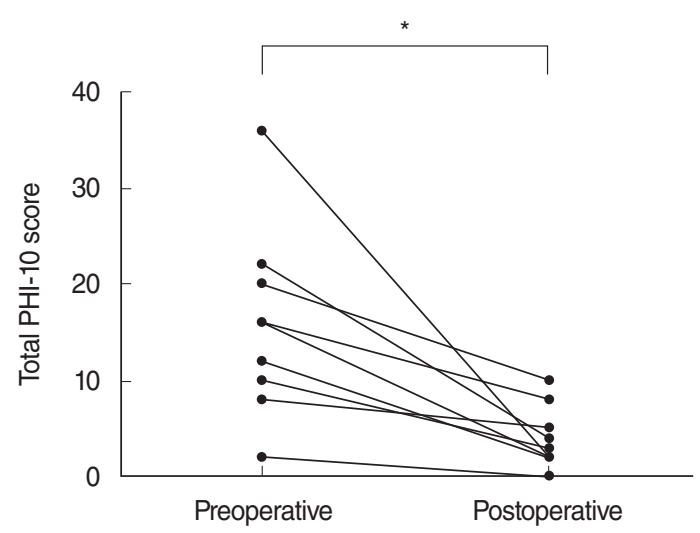

Fig. 5. Changes in total patulous eustachian tube handicap inventory-10 (PHI-10) score before and after the additional eustachian tube catheter insertion. ${ }^{\star} P<0.001$.

the inward and outward movement of the TM at the same time as forced nasal breathing. Patients consisted of nine pars flaccida type $(100 \%)$. Only one of nine sides had an air conduction hearing level worse than $60 \mathrm{~dB}$ in this study group. In contrast, eight of nine sides had a hearing level better than $60 \mathrm{~dB}$. One patient had bilateral cholesteatoma. Cholesteatoma surgery with additional ET catheter insertion was performed sequentially in both ears. Three patients (three ears) had the bilateral PET with unilateral cholesteatoma. The same procedure was performed for the cholesteatoma side; in addition, we inserted the catheter through the myringotomy site and placed into the bony orifice of the other ET as well. The average duration of preoperative habitual sniffing was 17.8 years (range, 10 to 30 years). Follow-up duration ranged from 16 to 37 months, with an average of 25.4 months. During an average follow-up of 25.4 months, no cholesteatoma recurrence has occurred to date. All patients had improved aural symptoms such as autophony, and stopped sniffing. Outcomes with respect to relief from autophony are presented in Table 3. One case developed otitis media with effusion (OME) after postcatheterization; OME resolved spontaneously without treatment. We confirmed good middle ear aeration at 3 months and 12 months postoperatively (Fig. 4). The preoperative and postoperative PHI-10 scores were $15.8 \pm 9.2$ and $4.1 \pm 3.0$, respectively $(P<0.001)$ (Fig. 5). According to outcome measurement for relief of PET symptoms after surgery, complete relief was reported for eight ears $(88.9 \%)$ and one ear $(11.1 \%)$ experienced significant improvement (Table 3).

\section{DISCUSSION}

In this study, we performed ET catheter insertion to partially occlude the ET simultaneously with cholesteatoma surgery (atticotomy or simple mastoidectomy) in patients with acquired cholesteatoma associated with PET and habitual sniffing. The average duration of preoperative habitual sniffing of the patient was 17.8 years (range, 10 to 30 years). We hypothesized that the only detailed explanation of their symptoms and mechanism of sniffing were not sufficient for these patients.

Our results showed that $88.9 \%$ of patients were satisfied with the complete relief of PET symptoms after surgery. However, autophony grade showed complete relief in $33.3 \%$ and significant improvement, satisfied in $66.7 \%$ of patients. The difference between the PHI-10 score and the autophony grade is thought to be because the PHI-10 score is not a simple questionnaire but the sum of scores for each of the 10 symptoms. Moreover, no cholesteatoma recurrence has occurred in any patient to-date. Patients stopped sniffing with an aerated middle ear without tympanic retraction. Otologic surgeons have occasionally observed retraction of tympanic membrane that caused recurrent cholesteatoma after surgery. In most cases, the mechanical or functional obstruction of the ET has been regarded as a major factor in the pathogenesis of retraction-type middle ear diseases. However, otologic surgeons must carefully evaluate the patients from another perspective in case of acquired cholesteatoma associated with PET and habitual sniffing. Contrary to the widely accepted concept that the pathogenesis of cholesteatoma is caused by ET dilatory dysfunction, it is important to note that, although rare, that habitual sniffing in patients with ET patulous dysfunction is closely related to the pathogenesis of cholesteatoma.

Management of middle ear cholesteatoma is mostly surgical and aims to make a safe ear without residual or recurrent cholesteatoma. Optimizing hearing and creating a non-discharging, self-cleaning ear are also important goals of cholesteatoma surgery [21]. However, most otologic surgeons mainly concentrate on eradicating pathologic lesions and overlook important points to eliminate the triggering factor. Previous studies have reported that habitual sniffing in patients with PET is closely related to the pathogenesis of cholesteatoma [1-12]. In 1996, Kobayashi et al. [9] first reported that patients with cholesteatoma had a significantly higher prevalence of habitual sniffing $(27.7 \%)$ than healthy subjects $(1.5 \%)$ and that habitual sniffing was found to be more common in the pars flaccida type (32.3\%) than in the pars tensa type ( $0 \%)$. Ohta et al. [10] reported that $25.7 \%$ of patients with cholesteatoma had a PET, and $27.4 \%$ of them were habitual sniffers. Ikeda reported that $23.4 \%$ of PET patients were found to possess evident sniffing habit and retraction-type middle ear diseases were significantly more common in PET patients with habitual sniffing $(53.6 \%)$ compared with PET patients without habitual sniffing (12.8\%) [1]. Habitual sniffing has been claimed to be a significant contributing factor to the development of postoperative retraction of the reconstructed posterior meatal wall and tympanic membrane [2]. Kawase et al. [22] reported severe retraction of the posterior meatal wall was found in seven of eight patients with habitual sniffing, but was significantly less (22 of 47) in ears without habitual sniffing. Yamamoto-Fukuda et al. [23] indicated that a tympanostomy tube was found to be effective in preventing the development of postop- 
erative retraction of the reconstructed soft posterior meatal wall and tympanic membrane among cases with habitual sniffing. These reports suggest that habitual sniffing and PET are influential cofactors in the pathogenesis of cholesteatoma and its recurrence. In audiologic aspect, according to the study of Hasegawa et al. [24], habitual sniffing was found in cholesteatoma patients with air conduction hearing level better than $60 \mathrm{~dB}$ or air-bone gap smaller than $40 \mathrm{~dB}$. As the habitual sniffing in patients with PET is usually a reaction to alleviate uncomfortable aural symptoms such as autophony and hyperacusis, the sniffing habit were dependent on hearing level. In this study, we confirmed eight of nine sides had a hearing level better than $60 \mathrm{~dB}$. In contrast, because of deteriorating hearing level, typical symptoms such as autophony and hyperacusis of PET are absent. Patients does not present with symptoms, but then complains of the symptoms after improvement of hearing [25].

However, there are no studies that propose a management protocol for cholesteatoma with PET and habitual sniffing. For management of such cases, understanding the mechanism of sniffing in PET patients is necessary. A previous study explained the mechanism as follows [1]: PET patients attempted to suppress auditory symptoms by a habitual sniffing, actively inducing a negative pressure in the middle ear to close the ET. Sudden negative pressure induced by sniffing causes blockage of the ET; this prevents vocalization sounds from reaching the middle ear directly via the ET. Patients obtain temporary relief from their symptoms when the ET is closed. However, since the ET easily opens after simple actions such as swallowing and yawning, so patients must sniff repeatedly to alleviate their symptoms [1]. Since habitual sniffing is considered to be an important initiator of acquired cholesteatoma, it is important for otologic surgeons to ascertain habitual sniffing in a patient before cholesteatoma surgery. Partial occlusion of the ET could help patients to reduce uncomfortable aural symptoms and stop habitual sniffing. Our postoperative results are consistent with these previous reports and further strengthen this hypothesis.

However, there are some limitations in this study. First, the number of cases identified (nine ears of nine patients) was low since acquired cholesteatoma associated with PET and habitual sniffing is an uncommon disease. Second, for similar reasons, there was no control group in which cholesteatoma surgery was performed without additional ET catheter insertion. Third, the patients underwent two different surgeries: cholesteatoma surgery (atticotomy or simple mastoidectomy) and ET catheter insertion. Accordingly, it is difficult to differentiate the effect of the two surgeries on the final treatment outcome. There is a possibility that the ear discomfort in general was improved by the cholesteatoma surgery (atticotomy or simple mastoidectomy). These limitations suggest the need for a well-designed, prospective, case-control study.

Habitual sniffing and PET may play a role in the pathogenesis and recurrence of acquired cholesteatoma. When otologic sur- geons decide to operate on cases with acquired cholesteatoma, the presence of habitual sniffing and PET must be evaluated. In case of acquired cholesteatoma associated with these issues, additional ET catheter insertion performed simultaneously with cholesteatoma surgery could be helpful in obviating habitual sniffing and preventing recurrence of cholesteatoma.

\section{CONFLICT OF INTEREST}

No potential conflict of interest relevant to this article was reported.

\section{ORCID}

Sung-Won Choi https://orcid.org/0000-0002-7463-7720

Dong-Joo Lee https://orcid.org/0000-0001-6219-2384

Seok-Hwan Lee https://orcid.org/0000-0002-5044-6735

Se-Joon Oh https://orcid.org/0000-0001-8910-0064

Soo-Keun Kong https://orcid.org/0000-0002-6783-3766

\section{AUTHOR CONTRIBUTIONS}

Conceptualization: SKK. Data curation: DJL. Formal analysis: SHL. Methodology: SWC. Project administration: SKK. Visualization: SJO. Writing - original draft: SWC. Writing - review \& editing: SKK.

\section{REFERENCES}

1. Ikeda R, Oshima T, Oshima H, Miyazaki M, Kikuchi T, Kawase T, et al. Management of patulous eustachian tube with habitual sniffing. Otol Neurotol. 2011 Jul;32(5):790-3.

2. Magnuson B. Tubal closing failure in retraction type cholesteatoma and adhesive middle ear lesions. Acta Otolaryngol. 1978 Nov-Dec; 86(5-6):408-17.

3. Magnuson B. On the origin of the high negative pressure in the middle ear space. Am J Otolaryngol. 1981 Feb;2(1):1-12.

4. Magnuson B. Tympanoplasty and recurrent disease: sniff-induced high negative pressure in the middle ear space. Am J Otolaryngol. 1981 Nov;2(4):277-83.

5. Falk B, Magnuson B. Evacuation of the middle ear by sniffing: a cause of high negative pressure and development of middle ear disease. Otolaryngol Head Neck Surg. 1984 Jun;92(3):312-8.

6. Falk B. Sniff-induced negative middle ear pressure: study of a consecutive series of children with otitis media with effusion. Am J Otolaryngol. 1982 May-Jun;3(3):155-62.

7. Kobayashi T, Takasaka T. Voluntarily retractable tympanic membranes: case report.Am J Otol. 1991 May;12(3):215-7.

8. Yaginuma Y, Kobayashi T, Takasaka T. The habit of sniffing in nasal diseases as a cause of secretory otitis media. Am J Otol. 1996 Jan; 17(1):108-10.

9. Kobayashi T, Yaginuma Y,Takahashi Y,Takasaka T. Incidence of sniff- 
related cholesteatomas. Acta Otolaryngol. 1996 Jan;116(1):74-6.

10. Ohta S, Sakagami M, Suzuki M, Mishiro Y. Eustachian tube function and habitual sniffing in middle ear cholesteatoma. Otol Neurotol. 2009 Jan;30(1):48-53.

11. Asawapittayanont P, Ikeda R, Oshima H, KikuchiT, Miyazaki H, Kawase $\mathrm{T}$, et al. Tubal function tests with optional myringotomy detect eustachian tube closing failure in acquired pars flaccida retraction cholesteatoma. Auris Nasus Larynx. 2017 Feb;44(1):65-9.

12. Tsuji K, Sone M, Kakibuchi M, Sakagami M. Bilateral cholesteatoma and habitual sniffing. Auris Nasus Larynx. 2002 Apr;29(2):111-4.

13. Oh SJ, Lee IW, Goh EK, Kong SK. Trans-tympanic catheter insertion for treatment of patulous eustachian tube. Am J Otolaryngol. 2015 Nov-Dec;36(6):748-52.

14. Sato T, Kawase T, Yano H, Suetake M, Kobayashi T. Trans-tympanic silicone plug insertion for chronic patulous eustachian tube. Acta Otolaryngol. 2005 Nov;125(11):1158-63.

15. Kikuchi T, Ikeda R, Oshima H, Takata I, Kawase T, Oshima T, et al. Effectiveness of Kobayashi plug for 252 ears with chronic patulous eustachian tube. Acta Otolaryngol. 2017 Mar;137:253-8.

16. Ikeda R, Kikuchi T, Oshima H, Miyazaki H, Hidaka H, Kawase T, et al. Relationship between clinical test results and morphologic severity demonstrated by sitting 3-D CT in patients with patulous eustachian tube. Otol Neurotol. 2016 Aug;37(7):908-13.

17. Kobayashi T, Morita M, Yoshioka S, Mizuta K, Ohta S, Kikuchi T, et al. Diagnostic criteria for patulous eustachian tube: a proposal by the Japan Otological Society. Auris Nasus Larynx. 2018 Feb;45(1): $1-5$.
18. Poe DS. Diagnosis and management of the patulous eustachian tube. Otol Neurotol. 2007 Aug;28(5):668-77.

19. Ikeda R, Kikuchi T, Oshima H, Miyazaki H, Hidaka H, Kawase T, et al. New scoring system for evaluating patulous eustachian tube patients. Otol Neurotol. 2017 Jun;38(5):708-13.

20. Tarabichi M, Najmi M. Visualization of the eustachian tube lumen with Valsalva computed tomography. Laryngoscope. 2015 Mar;125 (3):724-9.

21. Harris AT, Mettias B, Lesser TH. Pooled analysis of the evidence for open cavity, combined approach and reconstruction of the mastoid cavity in primary cholesteatoma surgery. J Laryngol Otol. 2016 Mar; 130(3):235-41.

22. Kawase T, Yuasa Y, Oshima T, Kobayashi T. Habitual sniffing and postoperative configuration of the posterior meatal wall reconstructed with soft tissue. Acta Otolaryngol. 2007 Nov;127(11):1132-5.

23. Yamamoto-Fukuda T, Takasaki K, Takahashi H. Effect of the tympanostomy tube on postoperative retraction of the soft posterior meatal wall caused by habitual sniffing. Laryngoscope. 2009 Oct;119 (10):2037-41.

24. Hasegawa J, Kawase T, Yuasa Y, HoriY, Sato T, KobayashiT. Effects of hearing level on habitual sniffing in patients with cholesteatoma. Acta Otolaryngol. 2006 Jun;126(6):577-80.

25. Kobayashi T, Hasegawa J, Kikuchi T, Suzuki T, Oshima T, Kawase T. Masked patulous eustachian tube: an important diagnostic precaution before middle ear surgery. Tohoku J Exp Med. 2009 Aug;218(4): 317-24. 